\title{
Tendência de resistência entre isolados clínicos de Staphylococcus aureus em um hospital universitário do norte do Paraná de 2002 a 2011
}

\section{Trends in antimicrobial resistance among clinical isolates of Staphylococcus aureus in a university hospital of the north of Paraná from 2002 through 2011}

\author{
Márcia Regina Eches Perugini ${ }^{1}$; Vitor Hugo Perugini²; Ana Rúbia Magalhães Ferreira ${ }^{3}$; \\ Caio Ferreira de Oliveira ${ }^{4}$; Guilherme Teixeira Gomes ${ }^{5}$; Bruna Arantes Rodrigues \\ Lima $^{6}$; Ana Paula Dier Pereira ${ }^{7}$; Floristher Elaine Carrara Marroni ${ }^{8}$; Eliana Carolina \\ Vespero $^{9}$; Marsileni Pelisson ${ }^{10}$; Maria Alice Galvão Ribeiro ${ }^{11}$
}

\begin{abstract}
Resumo
Staphylococcus aureus são patógenos com alta ocorrência em infecções hospitalares e comunitárias e têm grande capacidade de adquirir resistência. O objetivo deste estudo foi determinar o perfil de resistência aos antimicrobianos de $S$. aureus isolados no Hospital Universitário de Londrina de janeiro de 2002 a dezembro de 2011. A análise retrospectiva de 3.494 S. aureus foi realizada a partir de um banco de dados do setor de Microbiologia do Laboratório Clínico do Hospital Universitário de Londrina (HUL). Resistência aos antimicrobianos foi determinada de acordo com os critérios recomendados pelo Clinical Laboratory Standard Institute (CLSI-2011). Os maiores percentuais de resistência foram verificados para eritromicina $(49,4 \%)$, oxacilina, clindamicina $(41,8 \%)$ e ciprofloxacina $(36,5 \%)$. Adicionalmente, ocorreu redução significativa nas taxas de resistência à gentamicina, e a sulfametoxazol-trimetoprim. Todas as cepas analisadas foram sensíveis à linezolide. Verificou-se que $40 \%$ apresentaram susceptibilidade reduzida à vancomicina. Estes dados revelaram uma provável mudança na epidemiologia de S. aureus na nossa região, o que pode trazer impacto no tratamento e controle da infecção por este agente etiológico.
\end{abstract}

Palavras-chave: : Staphylococcus aureus. Resistência. Antimicrobianos. Meticilina.

\begin{abstract}
Staphylococcus aureus are pathogens with high incidence of nosocomial infections and community and has great ability to acquire resistance. The objective of this study was to determine susceptibility to antimicrobial profiles of S. aureus isolated in Londrina University Hospital, from January 2002 through December 2011. The retrospective analysis of 3,494 S. aureus performed from a database of Clinical Laboratory Microbiology HU sector. Antibiotic resistance was judged according to the criteria recommended by the Clinical Laboratory Standards Institute. The highest percentages of resistance was founding to erythromycin $(49.4 \%)$, clindamycin and oxacillin (41.8\%) and ciprofloxacin (36.5\%). In addition, there was a significant reduction
\end{abstract}

\footnotetext{
${ }^{1}$ Departamento PAC/CCS/Uel - Microbiologia.

${ }^{2}$ Discente do curso de Medicina/CCS/UEL - Doenças Infecciosas.

${ }^{3}$ Residente em Análises Clínicas/Uel.

${ }^{4}$ Mestre em Microbiologia/Uel.

${ }^{5}$ Residente em Análises Clínicas/Uel.

${ }^{6}$ Farmacêutica.

${ }^{7}$ Farmacêutica/Mestranda em Microbiologia/Uel.

${ }^{8}$ Farmacêutica/Doutora em Microbiologia/Uel.

${ }^{9}$ Farmacêutica/Doutora em Microbiologia/Uel.

${ }^{10}$ Farmacêutica/Mestre em Microbiologia/Uel.

${ }^{11}$ Graduação em Biomedicina pela Universidade Federal de Alfenas.
} 
in gentamicin resistance rates, and trimethoprim-sulfamethoxazol. All strains studied were susceptible to linezolid. We found that $40 \%$ showed reduced susceptibility to vancomycin. These data indicate a possible change in the epidemiology of $S$. aureus in our region, which can bring impact on the treatment and control of infection by this agent.

Keywords: Staphylococcus aureus. Resistance. Antimicrobial. Methicillin.

\section{Introdução}

As infecções hospitalares, atualmente, estão no centro das atenções quando o assunto é saúde pública. Isso se deve, tanto pelos graves desfechos que acometem os pacientes, como pelo enorme ônus gerado para assisti-los. Dentre os agentes responsáveis por tais infecções, Staphylococcus aureus é um dos mais frequentes. Trata-se de um coco gram-positivo de extrema relevância, capaz de causar infecções em indivíduos imunocomprometidos e sadios, associando-se às infecções tanto no ambiente hospitalar quanto comunitário (EUROPEANCENTRE FOR DISEASE PREVENTION AND CONTROL, 2013; ROSENTHAL et al., 2014, SIEVERT et al., 2013).

O sucesso terapêutico de infecções por $S$. aureus é comprometido, cada vez mais, pela multirresistência que este patógeno apresenta e pela sua capacidade de disseminação nos serviços de saúde, e mais recentemente na comunidade. Entre os antimicrobianos para os quais $S$. aureus apresentam resistência destacam-se betalactâmicos (MRSA-S. aureus meticilina resistente) e glicopeptídeos, (VISA-S. aureus com resistência intermediária à vancomicina e hVISA-S. aureus com resistência intermediária heterogênea à vancomicina (GALES et al., 2009; HIRAMATSU et al., 1997; HOWDEN et al., 2010; SADER; FLAMM; JONES, 2013).

Considerando a elevada frequência de $S$. aureus no Hospital Universitário de Londrina (HUL), e que o conhecimento da susceptibilidade aos antimicrobianos pode fornecer informações importantes para a implementação de terapia adequada e adoção de medidas de controle, o presente estudo teve por objetivo avaliar a tendência de resistência de $S$. aureus a antimicrobianos, num período de dez anos.

\section{Materiais e Métodos}

Trata-se de um estudo descritivo retrospectivo, no qual foi avaliada a frequência de resistência de $S$. aureus aos antimicrobianos, isolados de culturas de diversos materiais clínicos como, sangue, urina, pele e partes moles, secreções respiratórias, realizadas pelo setor de Microbiologia do Laboratório de Análises Clínicas do HUL, entre janeiro de 2002 a dezembro de 2011. Para tanto, utilizou-se o banco de dados do Sistema de Informação AGTA Healthcare, módulo LABHOS®, do LAC-HUL.

Aidentificação dos microrganismos foi realizada fenotipicamente, por metodologia manual, utilizando-se os testes de catalase, coagulase, DNAse, produção de hemólise e de pigmento, fermentação de manitol, e resistência a novobiocina, de acordo com metodologia padronizada por Versalovic et al. (2011) ou automatizada, utilizando-se os sistemas MicroScan ${ }^{\circledR}$ (Siemens Healthcare Diagnostic, Deerfield, IL.) ou Phoenix ${ }^{\circledR}$ (AB Biodisk, Solna, Sweden). A sensibilidade a antimicrobianos foi avaliada por metodologia manual de acordo com CLSI (2011) ou automatizada utilizando-se os mesmos equipamentos. Os antimicrobianos analisados foram ciprofloxacina, clindamicina, eritromicina, gentamicina, linezolide, oxacilina, penicilina, rifampicina, sulfametoxazol-trimetoprim e vancomicina. A partir de 2010 a susceptibilidade à vancomicina foi determinada também por microdiluição manual ou Etest ${ }^{\circledR}$ para110 amostras de MRSA obtidas de hemoculturas.

Este estudo foi aprovado pelo comitê de ética em pesquisa envolvendo seres humanos da Universidade Estadual de Londrina sob o CAAE número 0015.0.268.000-11. 


\section{Resultados}

S. aureus foi identificado em 3.494 culturas de materiais biológicos de pacientes internados ou atendidos no HUL. Foi o agente etiológico mais frequente em hemoculturas e o terceiro colocado entre todas as culturas analisadas no período de 2002 a 2011.

O perfil de resistência de $S$. aureus para 10 antimicrobianos é mostrada na tabela 1. Analisandose os betalactâmicos, verificou-se que para penicilina as taxas de resistência foram elevadas, tendo variado de 92\% em 2002 a 98\% em 2011. Já para oxacilina, o percentual médio verificado de cepas resistentes foi de $41,8 \%$, tendo sido evidenciando um aumento de resistência de 2002 (31\%) para 2011 (41\%).

Os antimicrobianos para os quais detectouse os maiores percentuais de resistência foram eritromicina, 52\% em 2002 a 46\% em 2011 (média 49,4\%), clindamicina 43 a $41 \%(41,8 \%)$ e ciprofloxacina 34 a $36 \%(36,5 \%)$.

Por outro lado, para e verificou-se redução significativa na frequência de resistência gentamicina de $36 \%$ para $15 \%$ e sulfametoxazoltrimetoprim de $31 \%$ para $10 \%$, de 2002 a 2011 , respectivamente.

Todas as cepas analisadas foram sensíveis a linezolide. Da mesma forma vancomicina apresentou $100 \%$ de sensibilidade pela metodologia de microdiluição automatizada. Entretanto, numa análise realizada posteriormente, com 110 amostras de $S$. aureus, isoladas entre 2010 e 2011, por microdiluição manual, verificou-se que $40 \%$ apresentavam sensibilidade diminuída à vancomicina ( $\mathrm{MIC}>2,0 \mu \mathrm{g} / \mathrm{mL}$ ).

Tabela 1 - Percentual de resistência a antimicrobianos (\%) de 3.494 amostras de $S$. aureus isolados de culturas realizadas no setor de microbiologia do HUL, no período de janeiro de 2002 a dezembro de 2011, por microdiluição automatizada MicroScan ${ }^{\circledR}$ ou microdiluição manual e Etest ${ }^{\circledR}$.

\begin{tabular}{|c|c|c|c|c|c|c|c|c|c|c|c|}
\hline & $\begin{array}{r}2002 \\
(\%)\end{array}$ & $\begin{array}{r}2003 \\
(\%)\end{array}$ & $\begin{array}{r}2004 \\
(\%)\end{array}$ & $\begin{array}{r}2005 \\
(\%)\end{array}$ & $\begin{array}{r}2006 \\
(\%)\end{array}$ & $\begin{array}{r}2007 \\
(\%)\end{array}$ & $\begin{array}{r}2008 \\
(\%)\end{array}$ & $\begin{array}{r}2009 \\
(\%)\end{array}$ & $\begin{array}{r}2010 \\
(\%)\end{array}$ & $\begin{array}{r}2011 \\
(\%)\end{array}$ & Média \\
\hline$N$ & 499 & 471 & 400 & 310 & 296 & 295 & 249 & 260 & 318 & 396 & \\
\hline $\mathrm{PN}^{*}$ & 92 & 98 & 99 & 98 & 97 & 98 & 97 & 98 & 98 & 98 & 97,3 \\
\hline OXA & 38 & 41 & 46 & 43 & 45 & 38 & 35 & 47 & 44 & 41 & 41,8 \\
\hline ERI* & 52 & 48 & 61 & 52 & 51 & 41 & 42 & 49 & 52 & 46 & 49,4 \\
\hline CLI* & 43 & 46 & 50 & 42 & 40 & 34 & 34 & 42 & 46 & 41 & 41,8 \\
\hline CIP* & 34 & 35 & 45 & 34 & 38 & 30 & 31 & 40 & 42 & 36 & 36,5 \\
\hline GN* & 36 & 37 & 45 & 36 & 39 & 26 & 29 & 33 & 26 & 15 & 32,2 \\
\hline $\mathrm{SXT}^{*}$ & 31 & 28 & 39 & 31 & 35 & 25 & 24 & 21 & 17 & 10 & 26,1 \\
\hline RIF* & 7 & 6 & 6 & 10 & 9 & 8 & 4 & 7 & 11 & 7 & 7,5 \\
\hline LZD* & 00 & 00 & 00 & 00 & 00 & 00 & 00 & 00 & 00 & 00 & 00 \\
\hline $\mathbf{V A N} \mathrm{N}^{*}$ & 00 & 00 & 00 & 00 & 00 & 00 & 00 & 00 & 00 & 00 & 00 \\
\hline $\mathbf{V A N} * *$ & NA & NA & $\mathrm{NA}$ & NA & NA & $\mathrm{NA}$ & NA & NA & 40 & 40 & 40 \\
\hline
\end{tabular}

N - Número de amostras testadas; CIP - ciprofloxacina; CLI - clindamicina; ERI eritromicina; GN - gentamicina; OXA - oxacilina; PN - penicilina; RIF - rifampicina; SXT - sulfametoxazol/trimetoprim; VAN - vancomicina. * avaliado por microdiluição automatizada, ** avaliado por microdiluição manual ou Etest ${ }^{\circledR}, \mathrm{NA}$ - não avaliado.

Fonte: autores 


\section{Discussão}

O presente estudo evidenciou uma tendência de aumento na resistência à oxacilina em $S$. aureus, bem como diminuição da sensibilidade à vancomicina nos últimos anos. A resistência a macrolídeos, lincosaminas, fluoroquinolonas e rifampicina se manteve estável em todo período, enquanto para gentamicina e sulfametoxazol-trimetoprim, evidenciou-se redução de resistência, em especial nos últimos anos.

Atualmente, $S$. aureus é o patógeno resistente a antibióticos mais comum em hospitais no mundo todo, tornando-se um problema clínico, de tratamento cada vez mais difícil. A alta prevalência e ampla disseminação de MRSA multirresistentes em hospitais brasileiros é uma preocupação no que se refere à segurança dos pacientes.

Variações importantes na prevalência de MRSA, de 1 a $61 \%$, têm sido evidenciadas por vários pesquisadores, em países de todo continente (EUROPEAN CENTRE FOR DISEASE PREVENTION AND CONTROL, 2013 ; ROSENTHAL et al., 2014; SADER et al, 2013; SIEVERT et al., 2013).

No Brasil a frequência de MRSA variou de 29 a $46 \%$, quando se analisam cepas de pacientes internados em hospitais, tanto com infecções hospitalares, quanto comunitárias. A frequência de MRSA verificada nesta pesquisa foi semelhante à de outros hospitais brasileiros (GALES et al., 2009; MARRA et al., 2011; SADER et al., 2013; SOUZA et al., 2014).

A proporção de MRSA em bacteremia aumentou significativamente nos anos 90, em várias partes do mundo. Entretanto, em países da Europa, a partir de 2010 a 2013, verificou-se uma tendência negativa no percentual de MRSA. No Reino Unido, entre 2001 e 2005, os índices de MRSA chegaram a 40-45\%, mas reduziram para $36 \%$ em 2007, após a implementação de estratégias de controle. Da mesma forma, na Espanha e Alemanha verificou-se redução das taxas de MRSA de $27,1 \%$ e $21 \%$ em 2005 para 22,6\% a 12,7\%, respectivamente. Em 2013, a média ponderada de MRSAna Europa manteve-se ao redor de $18 \%$, e sete dos 30 países que forneceram dados ao centro de Controle de Doenças da Europa (ECDC - European Centre for Disease Prevention and Control) apresentaram percentuais acima de $25 \%$ de MRSA (EUROPEAN CENTRE FOR DISEASE PREVENTION AND CONTROL, 2013).

A mesma tendência foi publicada pelo INICC, que evidenciou que entre $S$. aureus isolados de sangue, no período de 2004 a 2009, 84,4\% eram MRSA (ROSENTHAL et al., 2012) e de 2007 a 2012 eram $61,2 \%$, uma redução de cerca de 30\% (ROSENTHAL et al., 2014).

No estudo publicado por Villalobos Rodríguez et al. (2011), a frequência de MRSA se manteve estável no período de 2007 (38,2\%) a 2009 (36,8\%). Ao contrário, no Brasil, tem sido verificada uma tendência de aumento de MRSA. Teixeira e colaboradores (2012) verificaram aumento de MRSA de $23 \%$ em 1998, para $37 \%$ em 2008. No presente estudo o aumento de $30 \%$ verificado nas taxas de MRSA concordam com os demais estudos brasileiros. Esta diferença provavelmente seja atribuída a diferenças nas políticas de prevenção e controle destes microrganismos.

Resistência múltipla em $S$. aureus, além da resistência aos betalactâmicos, também tem sido reportada para outros antimicrobianos comumente utilizados como fluoroquinolonas, macrolídeos, lincosaminas, aminoglicosídeos, e sulfametoxazol-trimetoprim (GALES et al., 2009; HANAKI et al., 2014; SADER et al., 2013; TEIXEIRA et al., 2012).

O perfil de resistência a antimicrobianos tem sido relatado por diversos autores. Na publicação de Gales et al. (2009), os índices de resistência para 2.218 amostras de $S$. aureus, obtidos de vários materiais clínicos, entre 2005 a 2008 foram menores do que os do presente estudo. Para eritromicina verificou-se $38,1 \%$ de resistência, para clindamicina $28,4 \%$ para ciprofloxacina $29,9 \%$, ao passo que para sulfametoxazol/trimetoprim $24,5 \%$, foram semelhantes aos obtidos em 2002, mas superiores aos verificados em 2011. Quando avaliaram apenas as cepas de MRSA, entretanto, a frequência de resistência foi bem maior, eritromicina (94\%), clindamicina $(87,9 \%)$, ciprofloxacina $(91,4 \%)$ e sulfametoxazoltrimetoprim $(68,1 \%)$. 
Em outro estudo brasileiro, no qual foi avaliada a resistência para 104 MRSA isolados em hospitais, foi encontrada resistência elevada a eritromicina (100 e $100 \%$ ), clindamicina (100 e 96,4\%), ciprofloxacina (100 e 91,2\%) e gentamicina, nos anos 1998 e 2008, respectivamente. Outro fato observado foi a redução da resistência a sulfametoxazol-trimetoprim, de $100 \%$, em 1998, para 83,7\%, em 2008 (TEIXEIRA et al., 2012).

Pacheco et al. (2011) também encontraram índices elevados de resistência para isolados clínicos de MRSA em um hospital da cidade de São Paulo, $81 \%$ para eritromicina, $61 \%$ para gentamicina e $41 \%$ para clindamicina em isolados clínicos de MRSA. Os percentuais de resistência verificados por estes autores foram maiores do que os encontrados em nosso estudo, provavelmente pelo fato de terem sido incluídos apenas S. aureus resistentes a betalactâmicos, ao contrário do presente estudo, no qual avaliou a resistência de todos S. aureus isolados no período de estudo.

Em adição, tem sido observado em todo mundo, assim como no Brasil, a emergência de clones de MRSA que se apresentam sensíveis a vários antimicrobianos não betalactâmicos, em especial sulfametoxazoltrimetoprim (MATOS et al., 2014; MIRANDA et al., 2007; SCHUENCK et al., 2012; TRINDADE et al., 2005). Estas observações concordam com as realizadas no presente estudo, uma vez que a sensibilidade a sulfametoxazol-trimetoprim e gentamicina diminuíram 32 e 41\%, de 2002 a 2011, respectivamente. Esta redução provavelmente indica mudança clonal nos isolados do HUL, já que o clone epidêmico brasileiro, prevalente no Brasil até recentemente, apresenta resistência múltipla a antimicrobianos, com exceção de rifampicina e vancomicina.

No entanto, apesar de alguns destes compostos antigos, como sulfametoxazol-trimetoprim, exibirem boa atividade contra MRSA, assim como verificado em nosso estudo, a falta de dados de eficácia clínica, assim como a preocupação com a toxicidade destes medicamentos, impedem que sejam usados no tratamento de infecções estafilocócicas invasivas graves (CHAUDHARI et al., 2014; POGUE et al., 2011).
$\mathrm{O}$ antimicrobiano de escolha para tratamento destas infecções na atualidade é a vancomicina (HOWDEN et al., 2010). Entretanto, embora MIC $<2,0 \mu \mathrm{g} / \mathrm{mL}$ seja categorizada como sensível pelo CLSI (CLINICAL AND LABORATORY STANDARDS INSTITUTE, 2012), pesquisas indicam que MIC maior que $1,0 \mu \mathrm{g} /$ $\mathrm{mL}$ está associada a desfecho clínico desfavorável, colocando em risco a utilização deste medicamento.

Além disso, o uso excessivo de vancomicina acarretou o surgimento de resistência intermediária (VISA e hVISA) e alta resistência (VRSA) em várias partes do mundo (HIRAMATSU et al., 1997; LIMBAGO et al., 2014; MORAVVEJ et al., 2013; ROSSI et al., 2014).

A prevalência de hVISA varia amplamente de acordo com hospitais, regiões geográficas e populações de pacientes avaliados. Índices de 0 a 65\% têm sido reportados em vários estudos (HANAKI et al., 2014; HOWDEN et al., 2010).

Tal discrepância pode ser observada também no Brasil, onde relatos de VISA, embora ainda raros, variam de 0\% (GALES et al., 2009; ROSSATO et al., 2014) a 10\% (SILVEIRA et al., 2014), ao contrário do nosso estudo, que encontrou prevalência maior (40\%). Entre as possíveis razões para a divergência na frequência de hVISA estão a pequena frequência de células com sensibilidade diminuída e à variedade de metodologias para detecção de hVISA (HOWDEN et al., 2010).

Por outro lado, ao contrário da elevada frequência de hVISA, VRSA tem sido identificado raramente no mundo todo. Da mesma forma, nesse estudo, nenhum caso de VRSA foi identificado, assim como nos demais estudos brasileiros (GALES et al., 2009; ROSSATO et al., 2014; SILVEIRA et al., 2014). No Brasil, apenas um caso de alta resistência à vancomicina em $S$. aureus foi identificado em 2012, na cidade de São Paulo até o momento (ROSSI et al., 2014).

Outra opção terapêutica para cepas de MRSA é linezolide, uma oxazolidinona, que tem sido amplamente utilizada no tratamento de infecções por microrganismos gram-positivos resistentes à 
vancomicina. Embora resistência a linezolide em $S$. aureus (LRSA) (TSIODRAS et al., 2001) seja um problema emergente em várias regiões, incluindo Europa, América do Norte e América do Sul (GALES et al., 2006; GU et al., 2013), no presente estudo, este antimicrobiano apresentou excelente atividade contra $S$. aureus, uma vez que não foi identificado nenhum caso de resistência.

\section{Conclusões}

O presente estudo demonstra que a frequência de MRSA é elevada em nosso hospital e tem aumentado nos últimos dez anos. A resistência aos antimicrobianos comuns como macrolídeos, lincosaminas e fluoroquinolonas se manteve elevada em todo período. Por outro lado, a redução da sensibilidade a sulfametoxazol-trimetoprim e gentamicina nos últimos anos, sugere uma provável mudança no perfil epidemiológico de $S$. aureus no HUL. Este estudo evidencia que para linezolide, resistência ainda não foi relatada. Já para vancomicina não foi identificado resistência por metodologia convencional, mas quando se utilizou microdiluição manual ou Etest ${ }^{\circledR}$ verificouse frequência de VISA elevada em comparação aos demais estudos brasileiros.

Por fim, evidencia-se a necessidade de utilização de novas técnicas para avaliação da sensibilidade à vancomicinaedarealizaçãodetécnicasepidemiológicas com o objetivo de determinar a clonalidade de $S$. aureus e os mecanismos de resistência para subsidiar os clínicos no estabelecimento de terapia empírica e no controle de infecções relacionadas a este agente.

\section{Agradecimentos}

Agradeço ao Conselho Nacional de Desenvolvimento Científico e Tecnológico (CNPq) e Fundação Araucária pelo financiamento do presente estudo, à orientadora Márcia Regina Eches Perugini e aos funcionários do Departamento de Patologia e Análises Clínicas do HU/UEL.

\section{Referências}

CHAUDHARI, C. N.; TANDEL, K.; GROVER, N.; BHATT, P.; SAHNI, A. K.; SEN, S.; PRAHRAJ, A. K. In vitro vancomycin susceptibility amongst methicillin resistant Staphylococcus aureus. Med J Armed Forces India, New Delhi, v. 70, n. 3, p. $215-$ 9, Jul. 2014.

CLINICAL AND LABORATORY STANDARDS INSTITUTE. Performance standards for antimicrobial susceptibility testing: twentieth informational supplement. 2012. Disponível em: $<$ http://antimicrobianos.com.ar/ATB/wp-content/ uploads/2012/11/M100S22E.pdf $>$. Acesso em: 3 mar. 2015.

EUROPEAN CENTRE FOR DISEASE PREVENTION AND CONTROL - ECDC. Point prevalence survey of healthcare associated infections and antimicrobial use in European acute care hospitals. Stockholm: ECDC, 2013a.

Proportion of methicillin resistant Staphylococcus aureus (MRSA) isolates in participating countries in 2013. 2013b. Disponível em: <http://www.ecdc.europa.eu/en/healthtopics/ antimicrobial_resistance/database/Pages/graph_ reports.aspx>. Acesso em: 10 fev. 2015.

GALES, A. C.; SADER, H. S.; ANDRADE, S. S.; LUTZ, L.; MACHADO, A.; BARTH, A. L. Emergence of linezolid-resistant Staphylococcus aureus during treatment of pulmonary infection in a patient with cystic fibrosis. International Journal of Antimicrobial Agents, Oxford, v. 27, n. 4, p. 300, 2006.

GALES, A. C.; SADER, H. S.; RIBEIRO, J.; ZOCCOLI, C.; BARTH, A.; PIGNATARI, A. C. Antimicrobial susceptibility of gram-positive bacteria isolated in brazilian hospitals participating in the SENTRY Program (2005-2008). Brazilian Journal of Infectious Diseases, Salvador, v. 13, n. 2, 2009.

GU, B.; KELESIDIS, T.; TSIODRAS, S.; HINDLER, J.; HUMPHRIES, R. M. The emerging problem of linezolid-resistant Staphylococcus. Journal Antimicrobial Chemotherapy, Tokyo, v. 68, p. 4-11, 2013. 
HANAKI, H.; CUI, L.; IKEDA-DANTSUJI, Y.; NAKAE, T.; HONDA, J.; YANAGIHARA, K.; TAKESUE, Y. Antibiotic susceptibility survey of blood-borne MRSA isolates in Japan from 2008 through 2011. Journal of Infection and Chemotherapy, Tokyo, v. 20, n. 9, p. 527-34, Sep. 2014.

HIRAMATSU, K.; HANAKI, H.; INO, T.; YABUTA, K.; OGURI, T.; TENOVER, F. C. Methicillin-resistant Staphylococcus aureus clinical strain with reduced vancomycin susceptibility. Journal of Infection and Chemotherapy, Tokyo, v. 40, p. 135-136, 1997.

HOWDEN, B. P.; DAVIES, J. K.; JOHNSON, P. D. R.; STINEAR, T. P.; GRAYSON, M. L. Reduced vancomycin susceptibility in staphylococcus aureus, including vancomycinintermediate and heterogeneous vancomycinintermediate strains: resistance mechanisms, laboratory detection, and clinical implications. Clinical Microbiology Reviews, Washington, v. 23, n. 1, p. 99-139, 2010.

LIMBAGO, B. M.; KALLEN, A. J.; ZHU, W.; EGGERS, A. P.; LINDA, K.; MCDOUGAL, A.; VALERIE, S.; ALBRECHT, V. S. Report of the 13th Vancomycin-Resistant Staphylococcus aureus Isolate from the United States. Journal of Clinical Microbiology, Washington, v. 52, n. 3, p. 998-1002, Mar. 2014.

MARRA, A. R.; CAMARGO, L. F.; PIGNATARI, A. C.; SUKIENNIK, T.; BEHAR, P. R.; MEDEIROS, E. A. Nosocomial bloodstream infections in Brazilian hospitals: analysis of 2,563 cases from a prospective nationwide surveillance study. Journal Clinical Microbiology, Washington, v. 49, n. 5, p. 186671, 2011.

MATOS, P. D.; SEDACA, S.; FERREIRA, D. C.; IORIO, N. L.; TOLEDO, V. C.; FREITAS, A. I.; COELHO, F. L.; SOUSA, C.; DOS SANTOS, K. R.; PEREIRA, M. O. Antimicrobial synergism against different lineages of methicillin-resistant Staphylococcus aureus carrying SCCmec IV. Journal of Applied Microbiology, Orxford, v. 116, n. 6, p. 1418-26, 2014.
MIRANDA, O. P.; SILVA-CARVALHO, M. C.; RIBEIRO, A.; PORTELA, F.; CORDEIRO, R. P.; CAETANO, N.; VIDAL, C. F.; FIGUEIREDO, A. M. Emergence in Brazil of methicillin-resistant Staphylococcus aureus isolates carrying SCCmecIV that are related genetically to the USA800 clone. Clinical Microbiology and Infection, Paris, v, 13, n. 12, p. 1165-1172, Oct. 2007.

MORAVVEJ,Z.; ESTAJI, F.; ASKARI, E.; SOLHJOU, K.; NADERI NASAB, M.; SAADAT, S. Update on the global number of vancomycin-resistant Staphylococcus aureus (VRSA) strains. International Journal of Antimicrobial Agents, Netherlands, v. 42, n. 4, p. 370371, Oct. 2013.

PACHECO, R. L.; LOBO, R. D.; OLIVEIRA, M. S.; FARINA, E. F.; SANTOS, C. R.; COSTA, S. F.; PADOVEZE, M.C.; GARCIAC.P.; TRINDADE, P.A.; QUITÉRIO, L. M.; RIVITTI, E. A.; MAMIZUKA, E. M.; LEVIN, A. S. Methicillin-resistant Staphylococcus aureus (MRSA) carriage in a dermatology unit. Clinical Science, São Paulo, v. 66, n. 12, p. 2071-2077, 2011.

POGUE, J. M.; MARCHAIM, D.; KAYE, D.; KAYE, K. S. Revisiting "older" antimicrobials in the era of multidrug resistance. Pharmacotherapy, Malden, v. 31, n. 9, p. 912-21, Sep. 2011.

ROSENTHAL, V.D.; BIJIE,H.; MAKI,D. G.;MEHTA, Y.; APISARNTHANARAK, A.; MEDEIROS, E. A. International Nosocomial Infection Control Consortium (INICC) report, data summary of 36 countries, for $2004-$ 2009. American Journal Infection Control, St. Louis, v. 40, n. 5, p. 396-407, 2012.

ROSENTHAL, V. D.; MAKI, D. G.; MEHTA, Y.; LEBLEBICIOGLU, H.; MEMISH, Z. A.; ALMOUSA, H. H. International Nosocomial Infection Control Consortium (INICC) report, data summary of 43 countries for 2007-2012. Device-associated module. American Journal Infection Control, St. Louis, v. 42, n. 9, p. 942-56, 2014.

ROSSATTO, F. C.; PROENÇA, L. A.; BECKER, A. P.; SILVEIRA, A. C.; CAIERÃO, J.; D'AZEVEDO, P. A. Evaluation of methods in detecting vancomycin MIC among MRSA isolates and the changes in accuracy related to different MIC values. Revista do Instituto de Medicina Tropical de Sao Paulo, São Paulo, v. 56, n. 6, p. 469-72, 2014. 
ROSSI, F.; DIAZ, L.; WOLLAM, A.; PANESSO, D.; ZHOU, Y.; RINCON, S. Transferable vancomycin resistance in a communityassociated MRSA lineage. New England Journal of Medicine, Waltham, v. 17, n. 370, p. 1524-31, 2014.

SADER, H. S.; FLAMM, R.; JONES, R. N. Antimicrobial activity of daptomycin tested against Gram-positive pathogens collected in Europe, Latin America, and selected countries in the Asia-Pacific Region. Diagnostic Microbiology and Infection Disease, New York, v. 75, n. 4, p. 417-22, 2013.

SCHUENCK, R. P.; CAVALCANTE, F. S.; EMERY, E.; GIAMBIAGI-DE MARVAL, M.; DOS SANTOS, K. R. Staphylococcus aureus isolates belonging to different multilocus sequence types present specific virulence gene profiles. FEMS Immunology and Medical Microbioogyl, Amsterdam, v. 65, n. 3, p. 501-4, Aug. 2012.

SIEVERT, D. M.; RICKS, P.; EDWARDS, J. R.; SCHNEIDER, A.; PATEL, J.; SRINIVASAN, A. Antimicrobial-resistant pathogens associated with healthcare-associated infections: summary of data reported to the National Healthcare Safety Network at the Centers for Disease Control and Prevention, 2009-2010. Infection Control Hospital Epidemiology, Cambridge, v. 34, n. 1, p. 1-14, 2013.

SILVEIRA, A. C.; SAMBRANO, G. E.; PAIM, T. G.; CAIERÃO, J.; CORDOVA, C. M.; D'AZEVEDO, P. A. Is prediffusion test an alternative to improve accuracy in screening hVISA strains and to detect susceptibility to glycopeptides/lipopeptides? Diagnostic Microbiology and Infection Disease, New York, v. 79, n. 4, p. 401-4, 2014.

SOUSA, A. G.; DA COSTA, T. M.; CAVALCANTE, F. S.; CHAMON, R. C.; FERREIRA, D. C.; NOUÉR, S. A.; DOS SANTOS, K. R. Vancomycin minimum inhibitory concentrations using different susceptibility methods in Staphylococcus aureus isolates. Journal of Infection in Developing Countries, Italy, v. 8, n. 4, p. 558-60, 2014.
TEIXEIRA, M. M.; ARAÚJO, M. C.; SILVACARVALHO, M. C.; BELTRAME, C. O.; OLIVEIRA, C. C.; FIGUEIREDO, A. M.; OLIVEIRA, A. G. Emergence of clonal complex 5 (CC5) methicillin-resistant Staphylococcus aureus (MRSA) isolates susceptible to trimethoprim-sulfamethoxazole in a Brazilian hospital. Brazilian Journal of Medical and Biological Research, São Paulo, v. 45, n. 7, p. 637-43, Jul. 2012.

TRINDADE, P. A.; PACHECO, R. L.; COSTA, S. F.; ROSSI, F.; BARONE, A. A.; MAMIZUKA, E. M. Prevalence of SCCmec type IV in nosocomial bloodstream isolates of methicillinresistant Staphylococcus aureus. Journal of Clinical Microbiology, Washington, v, 43, n. 7, p. 3435-3437, 2005.

TSIODRAS, S.; GOLD, H. S.; SAKOULAS, G.; ELIOPOULOS, G. M.; WENNERSTEN, C.; VENKATARAMAN, L.; MOELLERING, R. C.; FERRARO, M. J. Linezolid resistance in a clinical isolate of Staphylococcus aureus. Lancet, London, v. 21, n. 358, p. 207-8, Jul. 2001.

VERSALOVIC, J.; CARRIOL, K.; FUNKE, G.; JORGENSEN, J. H.; LANDRY, M.; WARNOCK, D. W. Manual of clinical microbiology. 10th ed. Washington: ASM Press, 2011.

VILLALOBOS RODRÍGUEZ, A. P.; DÍAZ ORTEGA, M. H.; BARRERO GARZÓN, L. I.; RIVERA VARGAS, S. M.; HENRÍQUEZ IGUARÁN, D. E.; VILLEGAS BOTERO, M. V.; ROBLEDO RESTREPO, C. G.; LEAL CASTRO, A. L. Trends of bacterial resistance phenotypes in high-complexity public and private hospitals in Colombia. Revista Panamericana de Salud Publica, Washington, v. 30, n. 6, p. 627-33, Dec. 2011.

Recebido em: 05 ago. 2014. Aceito em: 03 mar. 2015. 